\section{Enteroliths in a Kock continent ileostomy: case report and review of the literature}

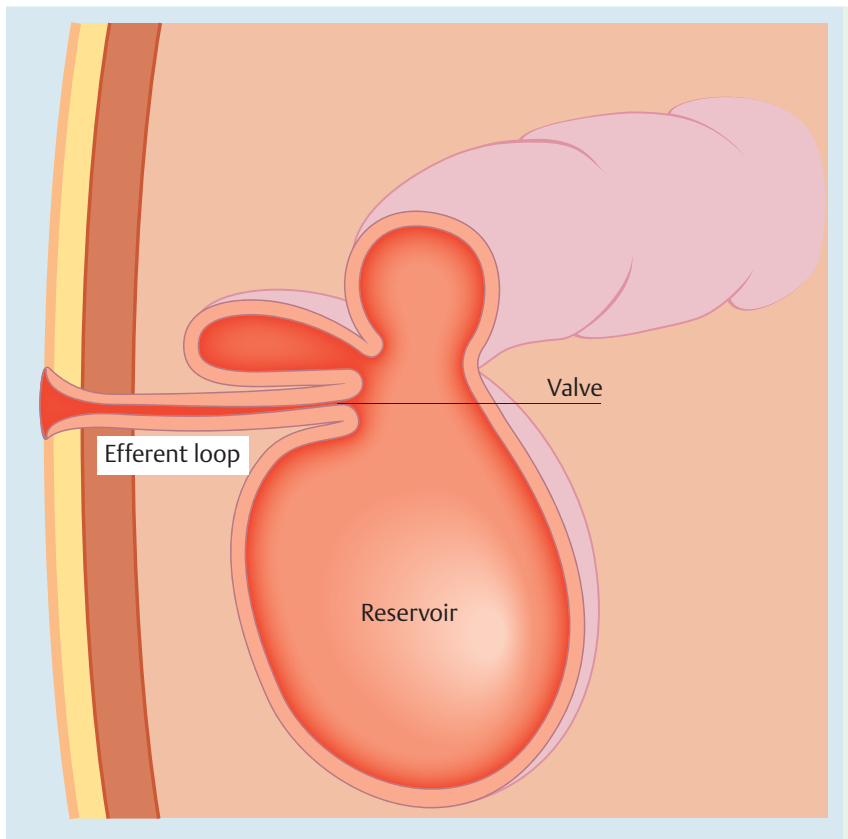

similar symptoms recurred 2 years later. A second ileoscopy showed a narrowed efferent loop that was dilated by insertion of the colonoscope, with successful relief of her symptoms. Chemical analysis of one of the retrieved enteroliths revealed calcium oxalate crystals. Five cases have previously been noted in the literature ( Table 1 ).

Fig. 1 Schematic representation of a Kock continent ileostomy.

The Kock continent ileostomy (KCI) was designed by Nik Kock, who used an intussuscepted ileostomy loop to create a nipple valve ( $\bullet$ Fig. 1 ) that would not leak and would allow ileal effluent to be evacuated with a catheter [1]. Enterolith formation is a rarely reported long-term complication of KCI that can lead to disabling symptoms mandating treatment [2-4].

We report the case of a 65-year-old woman who underwent total proctocolectomy and subsequent construction of a $\mathrm{KCI}$ when she was 31 years of age. The proce- dure was done to treat ulcerative pancolitis complicated by colon cancer. She had a well-functioning $\mathrm{KCI}$ that she had catheterized daily for 34 years before she presented with intermittent abdominal pain and occasional bleeding from the stoma, and she reported having difficulty catheterizing her ileostomy.

Computed tomography and ileoscopy demonstrated three oval enteroliths in the pouch and a lipoma in the efferent loop of the KCI ( Fig.2). The patient's symptoms decreased after resection of the lipoma with a snare cautery. However,
The alkaline milieu of succus entericus in the ileum may induce the precipitation of a calcium oxalate concretion; in contrast, the acidic milieu found more proximally in the intestine enhances the solubility of calcium. The gradual precipitation of unconjugated bile salts, calcium oxalate, and calcium carbonate crystals around a nidus composed of fecal material or undigested fiber can lead to the formation of calcium oxalate calculi over time [5].

Endoscopy_UCTN_Code_CCL_1AD_2AJ

Competing interests: None

\section{Hadi Moattar ${ }^{1}$, Jakob Begun, ${ }^{1,2}$ Timothy Florin ${ }^{1,2}$}

${ }^{1}$ Department of Gastroenterology, Mater Adult Hospital, South Brisbane, Australia ${ }^{2}$ Mater Research, University of Queensland, Translational Research Institute, Woolloongabba, Australia

\section{References}

1 Kock NG. Intra-abdominal "reservoir" in patients with permanent ileostomy. Preliminary observations on a procedure resulting in fecal "continence" in five ileostomy patients. Arch Surg 1969; 99: 223-231

2 Baig MK, Valerian B, Hakim L et al. Holmium: yttrium aluminium garnet laser lithotripsy for symptomatic Kock pouch calculi. Surg Innov 2006; 13: 193-197
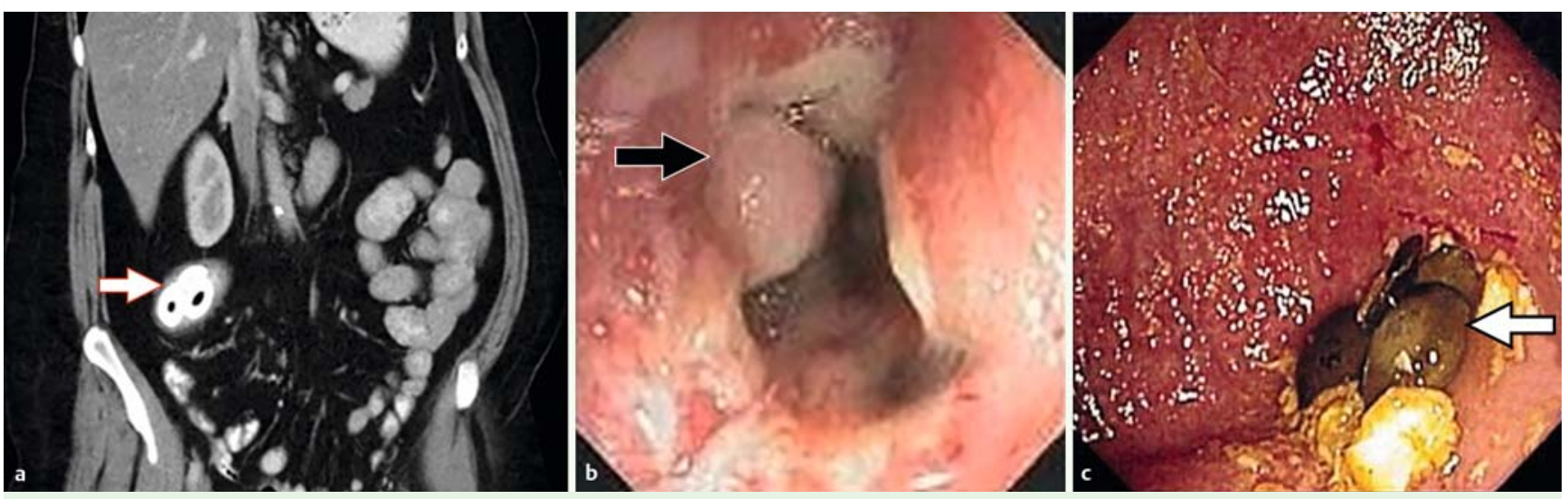

Fig. 2 a Abdominal computed tomographic scan demonstrating enteroliths in the Kock continent ileostomy (KCI) (arrow). b Polypoid lesion in the efferent loop of the $\mathrm{KCl}$ (arrow). c Darkly pigmented enteroliths in the $\mathrm{KCl}$ (arrow). 
Table 1 Reported cases of enterolith associated with Kock continent ileostomy (KCI).

\begin{tabular}{|c|c|c|c|c|c|c|}
\hline $\begin{array}{l}\text { Patient } \\
\text { gender } \\
\text { and age, } \\
\text { y }\end{array}$ & $\begin{array}{l}\text { Time } \\
\text { with } \\
\mathrm{KCl} \text {, } \\
\mathrm{y}\end{array}$ & Presenting symptoms & $\begin{array}{l}\text { Diagnostic modalities } \\
\text { Number of stones, } \\
\mathbf{n}\end{array}$ & Composition of stones & Treatment and outcome & Source \\
\hline Female, 48 & 23 & $\begin{array}{l}\text { Abdominal pain, } \\
\text { frequent need for pouch } \\
\text { catheterization }\end{array}$ & $\begin{array}{l}\text { Abdominal X-ray, } \\
\text { ileoscopy } \\
3\end{array}$ & $\begin{array}{l}\text { Calcium oxalate, } 95 \% \\
\text { Calcium phosphate, } 5 \%\end{array}$ & $\begin{array}{l}\text { Symptomatic relief after } \\
\text { endoscopic stone extrac- } \\
\text { tion following holmium- } \\
\text { yttrium-aluminum garnet } \\
\text { laser lithotripsy }\end{array}$ & Baig et al. [2] \\
\hline Female, 39 & 9 & $\begin{array}{l}\text { Increased pouch output, } \\
\text { abdominal cramps, } \\
\text { weight loss }\end{array}$ & $\begin{array}{l}\text { Abdominal X-ray, } \\
\text { barium study, ileoscopy } \\
15\end{array}$ & Calcium hydroxyapatite & $\begin{array}{l}\text { Symptom resolution after } \\
\text { surgical exploration and } \\
\text { stone removal }\end{array}$ & Fox et al. [3] \\
\hline Female, 53 & 20 & $\begin{array}{l}\text { Abdominal pain, } \\
\text { blood in pouch contents }\end{array}$ & $\begin{array}{l}\text { Abdominal X-ray, } \\
\text { ileoscopy } \\
30\end{array}$ & $\begin{array}{l}\text { Cholesterol, } 50 \% \\
\text { Other unidentified lipid, } \\
50 \%\end{array}$ & $\begin{array}{l}\text { Endoscopic extraction of } \\
\text { enteroliths with basket, } \\
\text { outcome not specified }\end{array}$ & Geller et al. [4] \\
\hline Male, 52 & 15 & $\begin{array}{l}\text { Abdominal pain, } \\
\text { blood in pouch contents }\end{array}$ & $\begin{array}{l}\text { Ileoscopy } \\
12\end{array}$ & Not specified & $\begin{array}{l}\text { Patient declined further } \\
\text { intervention }\end{array}$ & Geller et al. [4] \\
\hline Female, 55 & 13 & $\begin{array}{l}\text { Abdominal pain, } \\
\text { peristomal itching and } \\
\text { erythema }\end{array}$ & $\begin{array}{l}\text { Ileoscopy } \\
8\end{array}$ & Not specified & $\begin{array}{l}\text { Unsuccessful endoscopic } \\
\text { extraction with snare and } \\
\text { lithotripsy basket, patient } \\
\text { declined further interven- } \\
\text { tion }\end{array}$ & Geller et al. [4] \\
\hline Female, 65 & 34 & $\begin{array}{l}\text { Abdominal pain, } \\
\text { difficulty catheterizing } \\
\text { pouch, bleeding from stoma }\end{array}$ & $\begin{array}{l}\text { Abdominal computed } \\
\text { tomography, ileoscopy } \\
4\end{array}$ & $\begin{array}{l}\text { Calcium oxalate, } 90 \% \\
\text { Calcium phosphate, } 10 \%\end{array}$ & $\begin{array}{l}\text { Symptomatic improve- } \\
\text { ment after efferent loop } \\
\text { stricture relieved with } \\
\text { resection of a lipoma and } \\
\text { dilation }\end{array}$ & Current case \\
\hline
\end{tabular}

3 Fox ER, Chung T, Laufer I. Enteroliths in a continent ileostomy. AJR Am J Roentgenol 1988; 150: 105-106

4 Geller A, Clain JE, Lewis BS et al. Enteroliths in a Kock continent ileostomy: endoscopic diagnosis and management. Gastrointest Endosc 1998; 48: 306 - 308

5 Paige ML, Ghahremani GG, Brosnan JJ. Laminated radiopaque enteroliths: diagnostic clues to intestinal pathology. Am J Gastroenterol 1987; 82: $432-437$
Bibliography

Dol http://dx.doi.org/

10.1055/s-0034-1391302

Endoscopy 2015; 47: E200-E201

(c) Georg Thieme Verlag KG

Stuttgart · New York

ISSN 0013-726X
Corresponding author

\section{Hadi Moattar, MD}

Department of Gastroenterology Mater Adult Hospital

Raymoond Terrace South Brisbane Brisbane, Queensland 4101

Australia

Fax: +61731638548

hadi.moattar@mater.org.au 